\title{
Using Quality Standards in Evaluating the Performance of Small Private Hospitals and Achieving Patients' Satisfaction: An Exploratory Study in Jeddah, Saudi
}

\author{
Arabia \\ Nayef Salah Ghamri \\ Faculty of Economics and Administration King Abdulaziz University, Jeddah, Saudi Arabia \\ E-mail: nayef.alghamri@gmail.com
}

Received: March 2, 2019 Accepted: March 29, 2019 Published: April 19, 2019

doi:10.5296/bmh.v7i1.14447 URL: http://dx.doi.org/10.5296/bmh.v7i1.14447

\begin{abstract}
The current study reports on using quality standards namely, reliability, empathy, tangibility, and responsiveness in evaluating and improving the performance of small private hospitals in the Jeddah and their reflection on the level of the patients' satisfaction with the service provided. The research also concludes some findings and recommendations that may contribute to enhancing the service level in such hospitals and increasing the level of patients' satisfaction. Therefore, 121 questionnaires were distributed to patients in 15 small private hospitals in Jeddah city. A pilot stage was conducted on the questionnaire upon which, the 47 questions required for the final questionnaire were developed. The study shows that there is a strong positive statistical correlation between the five quality standards of medical services; reliability, empathy, confidence and assurance, tangibility and responsiveness and the level of patients' satisfaction with the hospital performance. The more patients are satisfied with the five quality standards, the higher the quality of medical services is. In addition, the study concluded a negative correlation between age and the level of patients' satisfaction with hospital services as elderly patients found to be less satisfied with the hospital services than younger patients. On the other hand, the hypothesis of having a statistical significant positive correlation between educational background and the level of patients' satisfaction with the hospital performance was refuted. Findings have shown a need for applying quality standards to improve the performance of small private hospitals and a necessity for working on overcoming the obstacles on their way.
\end{abstract}

Keywords: quality standards, hospitals, satisfaction, performance, patients, Saudi Arabia 


\section{Introduction and Theoretical Framework}

This research is made up of six parts:

1) Part I: Introduces the theoretical framework, problem, objectives, significance, and hypotheses.

2) Part II: Sheds light on previous studies.

3) Part III: Presents the research methodology, data collection techniques, data verification, and statistical methods employed.

4) Part IV: Displays the analysis of the results and tests the validity of the hypotheses.

5) Part V: Shows the results

6) Part VI: Concludes the recommendations of the study.

\subsection{Research Significance}

Hospitals, of all kinds and fields, play a significant role in health care due to their essential participation in treatment and health culture. This is in addition to their importance in social and training development. Needless to say, the quality of services plays an effective role in improving the performance of these hospitals.

\subsection{Research Problem}

Rapid changes in technology require hospital managers to keep pace with these developments so that they can improve the level of their services at the national and global levels. Therefore, these managers should be concerned with the development and research of the quality management systems which, in spite of being costly, are essential in hospitals, and they are becoming increasingly important in a rapidly changing competitive environment. The problem of research is that some hospitals, especially small private ones and polyclinics, continue to use the traditional culture of customer service, which negatively affects their position in the market and lacks access to information which lead to:

- Poor competitiveness.

- Lack of the use of quality standards systems which has resulted in hospitals not being able to access high quality service standards.

Quality standards systems of service delivery are considered of little use in small private hospitals. Consequently, It is believed that limited use of quality measurements has led to reduced quality of medical services and increased costs and length of waiting hours in these hospitals.

Thus, the problem of research lies in answering the following questions:

1) To which extent are service quality standards employed in small private hospitals in Jeddah, Saudi Arabia?

2) What is the role of using quality standards in improving the performance of small private 
hospitals and achieving patients' satisfaction?

\subsection{Research Objectives}

In addition to answering the research questions, the current study aims to:

- Show the importance of quality standards and their impact on business success.

- Urge small private hospitals to implement the systems of quality standards in their service processes via establishing a direct connection with customers' satisfaction.

\subsection{Hypotheses}

1) There is a statistical positive correlation between reliability, as one of the quality standards of medical services, and the level of patients' satisfaction with the hospital performance.

2) There is a statistical positive correlation between empathy, as one of the quality standards of medical services, and the level of patients' satisfaction with the hospital performance.

3) There is a statistical positive correlation between confidence and assurance, as one of the quality standards of medical services, and the level of patients' satisfaction with the hospital performance.

4) There is a statistical positive correlation between tangibility, as one of the quality standards of medical services, and the level of patients' satisfaction with the hospital performance.

5) There is a statistical positive correlation between responsiveness, as one of the quality standards of medical services, and the level of patients' satisfaction with the hospital performance. The more patients are satisfied with the five quality standards, the higher the quality of medical services is.

6) There is a statistical positive correlation between educational background and the level of patients' satisfaction with the hospital performance.

7) There is a statistical positive correlation between age range and the level of patients' satisfaction with the hospital performance.

\subsection{Independent Variables}

1) Achieving management's conviction which requires the commitment of the management, doctors and staff at all levels to apply the quality standards of medical service represented in (A) Responsiveness, (B) Reliability, (C) Assurance, (D) Tangibility, and (E) Empathy and to undertake appropriate activities and actions to meet these standards.

2) Staying away from the traditional organizational structure, reducing the influence of doctors, especially that most hospital managers are doctors, and paying more attention to the qualitative performance of doctors, rather than the quantitative performance that focuses on increasing income which may cause medical errors and low level of services in order to achieve a strong competitive environment (Srour, 2003). 


\section{Macrothink}

3) Enhancing the level of awareness, training and rehabilitation in order to achieve the dissemination of quality standards culture among doctors and administrators.

4) Using the technological tools and techniques as part of quality standards known as tangibility which requires encouraging workers to use technology to develop and improve the quality of medical services.

5) Encouraging employees' participation which requires the participation of every employee and doctor in every activity related to providing the best medical services. In addition to, encouraging them to make use of and benefit from their expertise and intellectual abilities in introducing any proposals that benefit the medical field and reduce the turnover rate among workers.

6) Building teamwork spirit among all hospital staff which contributes to improving the quality of services and enhancing the level of performance and effectiveness.

\subsection{Dependent Variables}

1) Reducing medical errors and waiting hours, and spreading the spirit of friendship between workers and patients, which includes the commitment of doctors and hospital staff to follow the quality standards of services.

2) Focusing on patients' satisfaction which means understanding their needs and expectations and providing such medical services that meet these needs and levels that the patients expect. Raising the morale of the patients and the level of efficiency of the hospital, and spreading loyalty culture among patients via the achievement of good relations between staff, at all levels in the hospital, and patients and providing patients the due care.

3) Contributing to reducing the cost of medical services, raising the level of efficiency and increasing the quantitative productivity via increasing the bed usage rotation rate and maintaining the reputation of the hospital in the medical field.

4) Improving competitiveness

\subsection{Introducing Hospitals}

Ancient civilizations used to consider illnesses and diseases as supernatural phenomena. They believed that certain types of food, plants and parts of animals could treat sickness. Indians had some medical wonders. For example, they found a relationship between malaria disease and mosquitoes. However, when it killed a quarter of the Europeans in the 1300s, it was necessary to investigate efficient scientific methods of treatment for such a medical catastrophe (Tiersky \& Tiersky, 1992). Meanwhile, Arabs were great in medicine as they had some well-known figures in medicine for instance Al-Razi, Ibn Sina, and others. They discovered several cures and had highly advanced medical instruments for surgeries. They used to treat their patients in hospitals. 


\subsubsection{Administrative Staff}

In the past, the administrator in a hospital used to have a clerical and a housekeeping role. As a result, their position was deemed lower than that of the medical staff. However, the role the hospital administrators play these days has changed dramatically thanks to the emergence of modern and sophisticated multi-unit hospitals and the necessity of dealing with the state-of-the-art medical equipment. Therefore, the administrator has started to have a pivotal role in coordinating the hospital day-to-day operations. This new powerful role could trigger conflict with the other two sources of power in the hospital.

\subsubsection{Authority Structure}

Hospitals do not often follow the same hierarchy system exhibited in other organizations due to the fact that there are three main sources of power, namely the board of trustees, administrators and the medical staff. There are usually some interferences between these three different sources of authority and none of them has central authority (Szilagyi \& Sims, 1974).

First of all, the board of trustees, which is an elected or appointed group of people with considerable expertise in management, tends to have a pure managerial role. They represent the hospital in the community and do their utmost to maintain its reputation. In addition, they provide the hospital with the needed resources for work. Nevertheless, they sometimes lack awareness of the daily operations of the hospital.

Second, hospital administrators play a strategic role of managing several departments in the hospital such as the purchasing department, the personnel department, the finance department, etc. They also manage the available health care resources and facilities and act as intermediaries and coordinators among the individuals within the organization. Administrators used to be doctors, yet recently degrees in hospital administration have been awarded to non-physicians by a number of universities around the world.

Last but, of course, not least, the medical staff is primarily in charge of patient treatment. It is worth mentioning that there have been calls to develop hospital administration professionally. Kast and Rosenzweig (1974) argue that such professionalization could be made possible through empowering efficient educational university programs, focusing on fulfilling the objectives of community service and activating the role of professional associations.

\subsubsection{Personnel and Services}

Christensen et al. (1982) posit that personnel salaries cost around 50 percent of a hospital budget, where nursing staff, on its own, costs 35 percent of the whole budget. Whereas, the other 50 percent serves for supplies, drugs, maintenance, taxes and interests. An average hospital has approximately twenty thousand items in stock.

\subsubsection{The Planning Function}

In hospitals, the planning function is shared between the medical staff and the administrator. The former performs the main role in relation to patient treatment and the latter plays a 
strategic role in terms of managing the available health care resources and facilities and behaves as an intermediary and a coordinator among individuals within the organization (Mankin \& Glueck, 1977). The two parties have to work collaboratively to ensure better and effective planning. Nonetheless, Kast and Rosenzweig (1974) state that the administrator will gain considerably increasing power in hospital planning, inter-hospital planning and community planning.

\subsubsection{Types of Hospitals}

There are various types of hospitals. The first type is the public hospitals in which governments usually offer the public a comprehensive free package of services such as precautions, vaccinations, control of infectious diseases, and family planning. In addition, there is also another type of hospital that does not charge patients; these hospitals are funded by charitable donations that is why they are called 'charity hospitals'. Moreover, there are other types of hospitals such as psychiatric or mental health hospitals, long-term acute-care hospitals, proprietary hospitals and 'specialist' hospitals for instance throat, nose and ear hospitals, children hospitals, eye hospitals, etc. More importantly, there is another type of hospitals which offers treatment to the patients and at the same time offers training to medical students, practitioners and nurses such as university medical hospitals.

\subsubsection{Hospital Operations}

Christensen et al. (1982) assert that hospital operations refer to managing the available resources for better patient outcomes. The services that hospitals offer could be divided into two categories: main and ancillary. The former includes room, board, housekeeping, medical and nursing services. However, the latter, ancillary, are those support services that include laboratory, respiratory therapy, pharmacy, electrocardiography, physical therapy services, ambulance, and emergency rooms. Finally, patients can be classified as inpatient and outpatient, where inpatient care requires at least overnight hospitalization.

\subsubsection{Inpatient Clinics}

These clinics take care of patients whose conditions require admission to a hospital for at least one overnight stay (Goh et al., 1999).

\subsubsection{Outpatient Clinics}

Outpatient clinics are responsible for the treatment of outpatients, i.e. patients with minor health problems who visit the hospital for quick treatment or diagnosis without needing to be admitted for overnight care.

\subsubsection{Emergency Department}

Emergency departments are in action around the clock to provide treatment to patients who are suffering an acute severe injury or illness that might cause serious health problems if not dealt with immediately. 


\subsubsection{Intensive Care Unit}

Intensive care units take care of patients who are suffering from life-threatening injuries or diseases such as patients with heart failure, heart attack and premature babies. Intensive care units provide ongoing care and close supervision through highly trained doctors and nurses who are specialized in dealing with patients in critical conditions.

\subsection{Introducing Quality and Its Standards}

Quality is not an invention or an innovation of the industrial revolution, but is, in fact, very old. One of the oldest concerns of the 18th century BC in the Babylonian civilization in Iraq in the laws of Hammurabi, which contained (282) articles, is the law on trade which obligates those who provide goods that are not good or worthless to repair the defect. The article number 229 of these laws states that "If a builder has built a house improperly for a man so that the house, he built, collapsed which caused the death of the landlord, the builder is to be killed" (Al-Ghezawi, 2002).

Prior to the development of the quality of services, from the time of products quality check up on the factory floor at the beginning of the 20th century to total quality management (TQM) at the early beginning of the twenty-first century, we note that skilled manufacturers were always responsible for the quality of the work they produced traditionally. This responsibility hasn't been transferred to the production line supervisor till the workers found themselves grouped with each other only in factories.

With the expansion of line supervisors' duties to include the recruitment, training and payment of workers at the end of the day, or week, and sometimes the settlement of disputes among them, the testing function has been introduced. In the North American factories, the testers checked the quality of all products before sending them to customers. This activity included raw materials received, as well as the parts between the processes. Then, testing, in turn, left the floor for monitoring so tests were designed, the control and monitoring systems were constructed and the laboratories were called into the scene.

At the end of the 1920s, statistical methods were developed and applied to quality control, including acceptance inspection and monitoring the process statistically. Monitoring is an activity aimed to detect errors and defects for the purpose of correcting and repairing them. However, prevention is more efficient and economical than the correction or repairing. Quality assurance has been provided and encouraged as suppliers were required to confirm to their customers that the products they ordered were designed, manufactured and delivered according to the required quality standards. In order for quality to be assured, it has to be planned and maintained in adequate organization (structures and systems); therefore, this shift from quality assurance to quality management has taken place. With the emergence of the total quality coding, awareness has been spread that there are standards that must be achieved and a new pattern of management should be adopted which is TQM.

TQM came to light with the Japanese innovation called quality circles, sometimes referred to as quality control circles. The aim of the quality circles was to have all employees meet in regular weekly meetings to discuss ways of improving the workplace and its quality, 
encourage employees to identify potential quality problems, and discuss and present their own solutions. Rapidly, the application of the TQM started to be the real criterion for the survival and growth of organizations. This paper summarizes topics related to the application of quality standards systems in order to build organizations in an interconnected, changing and competitive world.

Quality management is an ongoing process, and there is no time to end, because quality in its true sense is a comprehensive commitment to doing business properly to produce the best product and deliver outstanding service that meets the requirements of both the company and the customer.

The Japanese engineer, Taguchi and Konishi (1987) defined quality as an expression of the amount of avoidable loss that the product may cause to society after its delivery, including failure to meet customer expectations and failure to meet the performance characteristics and side effects resulting from its use on society such as pollution, noise and others. Williams (1999) defined quality as the suitability of the product for use which means its ability to deliver the best performance and the most authentic qualities. Khodeir (2000) believes that quality consists of four main axes:

- The company may use the right items incorrectly. They may have modern sophisticated medical equipment, but they do not use them properly, or the technicians at this facility may misuse them.

- The establishment may have an inefficient scheduling system, and staff at reception offices may not be well trained on the system, so the result will be unsatisfactory and therefore this will reflect a poor quality of the service provided.

- On the other hand, the situation may be versed which means wrong things may work correctly. The establishment may have an inefficient scheduling system, but staff do their job well when scheduling patients so they fix that being clever enough to make it work.

- Finally, high quality lies in doing the proper and right things appropriately and in the right way. For example, a facility has developed medical equipment, and staff use them well, reflecting a high degree of effectiveness and efficiency, which reflects well on the organization financially, administratively and technically.

Thus, we can conclude that the definition of quality is so multifaceted that it cannot be confined to a narrow circle. It takes different dimensions that include technical, administrative, behavioral, social and clinical concepts. Garvin (1988) divided the concept of quality into several types as follows:

- Excellence-based approach: this means that although the individual can distinguish between high and poor quality, there is no specific definition of quality. For example, we cannot say that the cardiologist is a skilled doctor or has high quality in his own clinical skills unless he performs a number of successful surgeries.

- Product-based approach: this approach identifies some factors or characteristics that can be measured to illustrate the high quality level. A digital medical device, for example, 
may be evaluated as better than a standard medical device.

- Beneficiary-based approach: this approach is one of the most important approaches that define the concept of quality. The definition of quality depends on the satisfaction of the customer with the goods or services provided and the extent to which these goods and services meet the client's wishes, needs and expectations. Through this approach, quality is defined on the basis of conformity of goods and services to a set of predefined requirements, so the customer will be satisfied when the components of the item or the characteristics of the service conform to the requirements. In the case of non-conformity, this will resemble a kind of defect and deviation in the product or service, and thus will negatively affect the level of the customer satisfaction.

- Value-based approach: the definition of quality as linked to the price so that quality is defined as the degree of excellence at the lowest prices, as well as the extent to which defects are monitored at the lowest costs.

As per Allam and Shohaib (2001) the quality sought by clients is best to include what they want and expect according to the level of service as well as the level that removes their reasons for complaining and dissatisfaction. Hence, Al-Saqaf (2001) believes that reinforcing the quality of the production process shall include the design and implementation of the service delivery procedures so as to ensure that the resources and capabilities available are utilized as efficiently as possible, provided that they meet the basic needs and requirements of the customers. It is also important to establish the rules of values and benefits shared by employees and increase their awareness of the importance of quality in general, and the importance of loyalty and belonging to the objectives of the establishment in particular, as well as dealing with each individual in the establishment through the impression of not accepting any less than the highest level of quality. Moreover, employees should be familiarized with the concept of quality and encouraged to try to understand this concept, taking into account their suggestions and opinions regarding continuous improvement. Needless to say, staff should be trained in simple statistical methods in an effort to identify, analyze and overcome quality problems.

It is clear from the above that quality is the main axis of the service and productivity process. It is possible that some goods are of good quality at present, but time can change the perception through the development of people's tastes as the change in quality continues through the competition of the rival parties to develop and improve the quality of their products.

\subsubsection{Development of Medical Quality Concepts in Hospitals}

There are several stages to develop the concept of quality as follows:

1) Quality of medical service delivery which includes:

- Minimizing medical errors.

- Measuring the cost of medical errors. 


\section{Macrothink}

- Stimulating hospital staff to comply with quality requirements.

2) Satisfying the client (patient) which includes:

- Building close relation with the patient.

- Understanding patient needs and expectations.

- Making most hospital decisions based on the wishes of the patient.

3) Quality as a factor in medical competition which includes:

- Introducing the medical market as the basis of all decisions.

- Identifying competitors and trying to outdo them.

- Finding out the reasons why patients are not dealing with the hospital.

Ishikawa (1990) summarizes the basic principles of quality control as follows:

1) Quality is based on the customer's point of view.

2) Quality is the essence of the management process, preferably as a long-term investment rather than focusing on profits in a short period of time.

3) Quality depends entirely on the active participation of staff. Besides, the implementation of quality management requires the removal of barriers and having harmony among different departments.

\subsubsection{Quality Standards}

Goods have eight standards of quality as follows:

- Performance: the way of performing and processing the function.

- Appearance: the tangible characteristics, shape, sense and brilliance of the commodity.

- Reliability: performance of the required tasks under specific operational conditions over a period of time.

- Conformance: compliance with specifications specified by contract or by customer.

- Durability: comprehensive and durable utilization of goods.

- Serviceability: ability to be modified or repaired when there is a problem in using it as a result of a problem in its manufacturing (Al-Shenwani, 1996).

- Aesthetics: the brilliance, shape and sense that the goods emphasize.

- Perceived Quality: the customers' perception about the goods and its ability to meet their needs regardless of its actual quality.

Service has eight standards of quality as follows:

- Time: the customer's waiting time 
- Timeless: delivery on time

- Committers: completion of all the service aspects in full

- Courtesy: welcoming all customers

- Consistency: delivery of all services in the same style and manner to the customer

- Convenience: Easiness of having access to the service

- Accuracy: providing the service correctly since the first moment

- Responsiveness: the time needed to give feedback or meet the customer's needs

\subsubsection{Medical Services Quality Standards}

A. Tangible aspects as defined by Wilson and Zeithaml (2012), include the design and internal organization of buildings, modern equipment and medical devices and the appearance of doctors and employees.

B. Empathy as explained by Shaikh and Rabbani (2005), include the necessity of understanding the needs of the patients and placing their interest as a priority for the management and employees, maintaining appropriate working hours and time for the service provided, paying personal attention to each patient and putting the patient's condition into consideration, showing empathy with the patients, and dealing with them friendly with humor.

C. Assurance as per Thamer (2005), include the sense of security in the treatment, knowledge and skill of specialized doctors, politeness and good attitude of the staff, the continuity of follow-up on the patient status, confidentiality of the patient's information, and the support of management for the staff to perform their duties efficiently.

D. Responsiveness as stated by Hamid (2008) and El Masry et al. (2015), in the field of medical services, include speed in providing the required medical service, immediate response to the needs of the patient regardless of the degree of being busy, the constant readiness of the staff to cooperate with the patient, the immediate response to the patient's inquiries and complaints, and as added by Al-Ghezawi (2002) informing the patient exactly when the service will be provided and accomplished.

E. Reliability, in the field of medical service as Sekaran (2003) and Slack et al. (2010) believe, means adherence to the scheduled appointments of the beneficiaries (patients), as well as the delivery of the results of laboratory tests and scans to the beneficiaries according to the scheduled dates, accuracy and error-free examination or diagnosis or treatment, availability of different specialties, confidence in doctors and specialists, care to solve the patient's problems, and keeping accurate file records.

Some of the above mentioned medical service dimensions of quality can be assessed objectively while others will remain subject to personal views and cultural, educational, and economic backgrounds. While the patient's satisfaction is the sense of accepting the service provided by the hospital to the patients and their belief in the attention and care paid by the 
medical staff, which creates a sense of loyalty in the future towards this hospital. This care includes the attention paid by administrators to the humane and good treatment of patients which requires a rapid response to the patient's demands and the continuous improvement of the provided services. Al Faraj (2009) classified the medical services into two types, preventive or protective and therapeutic Services. The preventive or protective services are the ones that protect the human health against disease. Whereas the therapeutic services helps in getting rid of the disease after its occurrence through examining the patient, diagnosing his illness, admitting him to one of the therapeutic institutions, and providing him the medicine and food appropriate to his case as well as dealing with him well in order to help him recover quickly.

\section{Review of Literature}

The study of Sreenivas and Prasad (2003) showed that the patient expects the availability of three basic factors during his stay at hospital: comfort, care and treatment. Thus, his study identified possible factors that affect the level of patients' satisfaction in hospital as follows:

- Reliability which is the ability of the hospital to perform the services expected by patients accurately and perfectly.

- $\quad$ Response speed which means the desire of workers to help patients, and provide service quickly when needed.

- Employees' behavior which means that the staff are courteous with decent behavior when communicating with patients which helps to give the patient confidence.

- Attention which means that employees show an evident willingness to show empathy and care to patients individually.

- Hospital structure which refers to the physical appearance of the hospital, as well as equipment, materials and means of communication available and showing them in the proper required shape.

Similarly, Jawahar (2007) explained that patients' satisfaction is an important variable in the highly competitive health care market. The study was conducted in order to determine the level of patients' satisfaction by getting feedback from them about the services provided in the outpatient clinics department of the hospital. The results of the study showed that $90-95 \%$ of the 200 patients surveyed were satisfied with the level of service in the hospital. This study also showed that the waiting time was very long and that the behavior of hospital staff needs to be improved with respect to the courteous and decent dealing with patients. This study considered several variables such as waiting time, hospital facilities, employees' performance, scheduling appointments system, and employees' behavior.

Furthermore, numerous studies were conducted in Jordan, including the study of Al-Edwan (1996) on the satisfaction of citizens with the public services provided by the governmental departments, ministries and service institutions in Jordan. These included health care services and concluded that the residents of the villages were more satisfied than the urban population. In addition, there is a statistical significant positive relation between age, qualification and 
the nature of the profession of the beneficiary and their satisfaction with the provided services. Again with Al-Edwan and Ahmed (1997) in their study which aimed to identify the most important factors related to the satisfaction of patients residing in Jordanian hospitals, they found out that satisfaction is related most importantly to factors such as: hospital cleanliness, health insurance availability, doctor's attention during treatment, and the type of hospital.

In the same vein, Cleveland Clinics, in 2002, launched an unprecedented World Class Service program to promote a world-class service culture among its employees and motivate them to provide feedback and suggestions for improving service, quality, growth and profits. Through this program, Cleveland clinics have expressed their goal of improving the level of satisfaction of their patients in proportion to their reputation and medical achievements. Likewise, focusing on patients' satisfaction with the medical service and the factors that may limit citizens' access to primary health care, Abdulqader (2015) concluded that the distance travelled by the patients to the hospital affects the level of their satisfaction with the service.

In another study by Cheng and Tang (2000) which compared patients' expectations and their impressions of the services provided and analyzed them. The study included 252 patients in Singapore hospitals. This study showed a gap between the patients' expectations in terms of the quality of health services and their impressions after benefiting from them. The study recommended the necessity of incorporating the quality assurance process into the tangible aspects of services, responsiveness, empathy, care, ease access of service, price suitability and insurance. In addition, Matarid (1999) examined the evaluation of the quality of health services in the investment hospitals and found that there is a decline in the level of health services and inability of the hospitals to evaluate their performance and recommended the adoption of customer expectations as strategies to achieve the best returns. It also recommended the implementation of quality control systems, commitment to training the medical, technical and administrative staff, and the effective application of information systems to facilitate administrative decision-making.

Al-Hadad (2006) focused in his study on finding out the reality of the practice of the department managers of planning in hospitals and unraveling the relationship between such a practice and the quality of health services in the hospitals under study. The study revealed that there is a lack of administrative experience among officials who are always busy with the therapeutic tasks directly which resulted in shortcomings in the quality level of health services. It has also been concluded that the leaders are doctors, not administrative and accounting specialties which led to a low level of planning due to the lack of qualified manpower. Along the same line, Al-Ahmadi (2000) shed light in her study on the organizational crisis using the approaches of thought and the administrative and organizational approach to determine its causes and how to reform it. The study concluded that the external environment and management have a direct role in exacerbating the problem. In addition, the conflict of objectives and interests, poor management skills, mismanagement, lack of resources, the incompatibility of powers and responsibilities, the weakness of the followed policies are of the direct reasons causing the organizational crisis. The study recommended addressing the external environmental problems and organizational factors mentioned in the study. 
Nosairat and Al-Domour (2000), in their study about the quality components of hospital services, aimed to identify the differences and the extent of variation between hospital management and the patient population based on some demographic characteristics of each of the two groups. The study found a significant difference in the relative importance given to the quality components and found similarity between the two groups in the relative importance given to the component of human interaction. The study also found a significant difference between patients on the relative importance of perceived quality components when classified according to income or educational level. The study also showed the importance of patient satisfaction, which is indispensable when providing the medical services, assessing the quality, planning and managing such services.

\section{Methodology}

The current study employs the analytical descriptive and applied approaches. The analytical descriptive approach depends on access to Arabic and Western references, internet sites, periodicals and research that preceded in this field. The applied approach depends on designing a questionnaire containing 47 Likert scale questions and distributing it to the sample of the research. Then, collecting, defining, and analyzing data to reach the findings and recommendations for the subject of research.

\subsection{Sampling and Data Collection}

A random sample of 121 patients has been employed voluntarily in this study. A random sample of 15 small private hospitals is selected to determine the extent to which quality systems are being used and their impact on the performance of these hospitals. The sample is restricted to small private hospitals in Jeddah. The questionnaire has been distributed to the patients to determine the extent to which the quality standards of health services in these hospitals are being used and the obstacles to their application.

\subsection{Study Tools}

Statistical Analysis Methods: IBM SPSS 21 software has been employed in the statistical analysis of data. Statistical measures, such as frequencies, percentages, Cronbach's alpha test, Kolmogorov-Smirnov and Shapiro-Wilk tests, Spearman's Rank Order Correlation (rho) have been used.

\subsection{The Time Limit}

Reviewing books and journals, searching the Internet for data, distributing and analyzing questionnaires and drafting the research paper took about a year.

\subsection{The Spatial Limit}

The current study has been conducted in Jeddah governorate in the Western Region of Saudi Arabia.

\subsection{Challenges}

Patients' lack of interest in the research in general, as some of them did not give enough time 


\section{Macrothink}

to complete the questionnaire properly and even sometimes they declined to participate in the study.

\section{Data Analysis}

\subsection{Instrument}

Data were collected through a 47-item questionnaire on a 5-point Likert scale, where $5=$ strongly agree and $1=$ strongly disagree. Two hundred copies of the questionnaire were distributed, but only one hundred and forty-three copies were returned. After revising the returned questionnaires and removing the improperly filled out ones, only 121 questionnaires were analyzed with a $60 \%$ response rate. The data collected were coded, entered and processed using IBM SPSS Statistics 25 . The survey had one negatively worded item (item number 23) which was reversed before scoring. To make sure that the data set was error free before proceeding with data analysis, checks for outliers, i.e. values that are well below or above the other scores, were conducted (Crowder, 2017). One data entry error was found and corrected. Statistical measures, such as frequencies, percentages, Cronbach's alpha test, Kolmogorov-Smirnov and Shapiro-Wilk tests, Spearman's Rank Order Correlation (rho) were carried out to fulfill the objectives of the study.

\subsection{Participants}

The demographic profile of the recruited participants includes four variables, namely gender, age, qualifications, and years of experience. The total number of the participants is $\mathrm{n}=121$. The descriptive analyses indicate that $69 \%$ of them are male (84 participants) and $31 \%$ of them are female (37 participants). As for their ages, Table 1 below illustrates that the majority of them $(69.5 \%)$ are 40 years old or less, whereas $(30.5 \%)$ of the participants are above 40 years of age.

Table 1. Ages of the Participants

\begin{tabular}{llll}
\hline Age & & Frequency & Percent \\
\hline Valid & less than 30 years & 44 & 36.4 \\
& $31-40$ years & 40 & 33.1 \\
& $41-50$ years & 17 & 14.0 \\
& $51-60$ years & 20 & 16.5 \\
& Total & 121 & 100.0 \\
\hline
\end{tabular}

As per the participants' educational qualifications, Figure 1 below shows that the majority of participants $(43 \%)$ are bachelor degree holders, $(35.5 \%)$ of them have masters or higher degrees, and $(21.5 \%)$ of them have only high school certificates. 


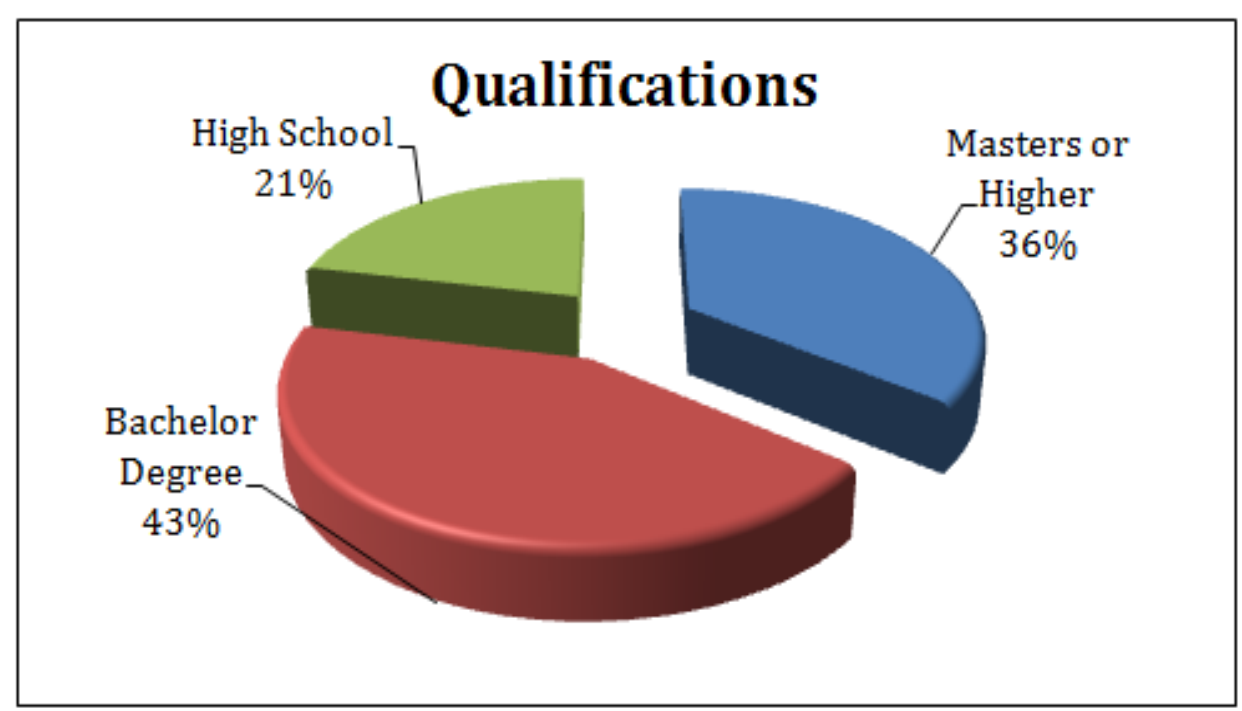

Figure 1. Description of the Sample According to Their Educational Qualifications

With reference to the participants years of work experience, Figure 2 reveals that the majority of the respondents (59\%) have less than five years of work experience, $17 \%$ of them have 6 to 10 years, $11 \%$ have 11 to 20 years, $9 \%$ have 21 to 30 years, and finally only $4 \%$ of the participants have 31 to 40 years of experience.

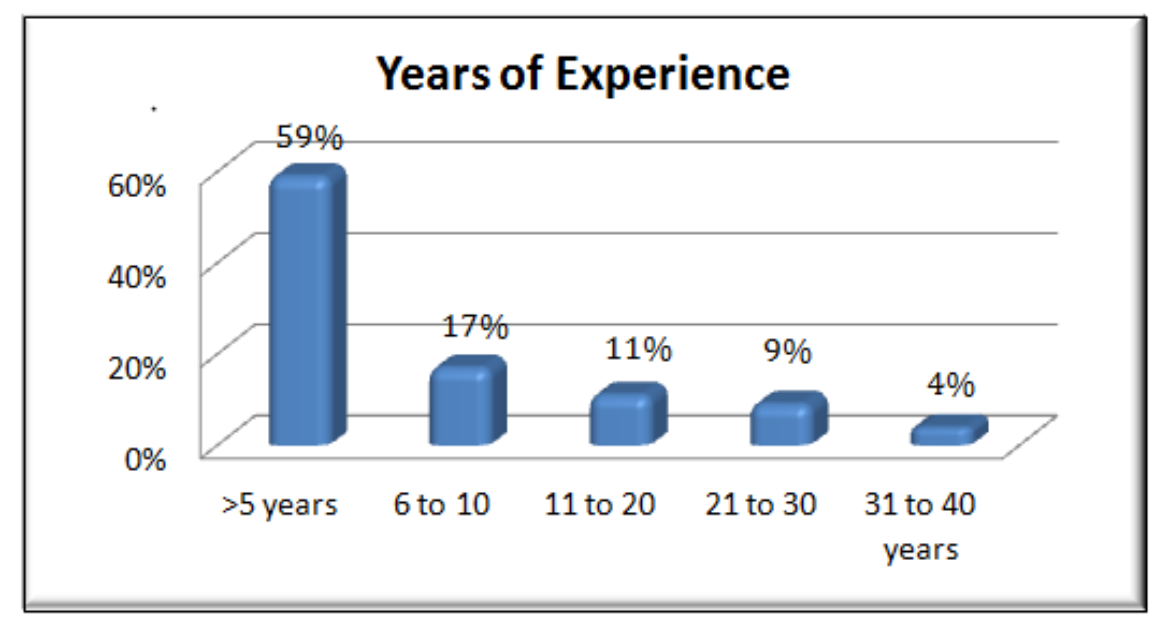

Figure 2. Distribution of the Participants According to Years of Work Experience

\subsection{Reliability of Data Collection Instrument}

Since the instrument was developed by the researcher specifically for this study, the reliability of the instrument had to be investigated. The reliability of an instrument refers to how free it is from random error. Internal consistency is commonly used to measure a scale's 
reliability (Taber, 2018). Therefore, Cronbach's Alpha Coefficient analysis of the five constructs in the survey was carried out. Table 2 below highlights the Cronbach's Alpha Coefficient for each construct.

Table 2. Cronbach's Alpha Coefficients for the Five Constructs of the Instrument

\begin{tabular}{|c|c|c|c|c|}
\hline No & Construct & Items & Number of Items & Cronbach's Alpha \\
\hline 1 & Tangibility & $1,2,3,4,5,6,7,8 \& 27$ & 9 & .902 \\
\hline 2 & Reliability & $9,10,11,12,14,22,23,24,25,26 \& 31$ & 11 & .872 \\
\hline 3 & Responsiveness & $13,15,18,19,20 \& 21$ & 6 & .891 \\
\hline 4 & $\begin{array}{l}\text { Confidence \& } \\
\text { Assurance }\end{array}$ & $16,17,28,29,30,32,33,34,35,37,38 \& 39$ & 12 & .912 \\
\hline 5 & Empathy & $36,40,41,42,43,44,45,46 \& 47$ & 9 & .922 \\
\hline
\end{tabular}

As exhibited in Table 2, the analyses revealed high levels of internal consistency in each construct of the instrument. Thus, the instrument has yielded reliable raw data.

\subsection{Normality and Correlation Tests}

Normality of distribution for each construct of the study was checked using the Kolmogorov-Smirnov and Shapiro-Wilk tests. As shown in Table 3 below, the results indicated that all the five constructs were not normally distributed at $p<0.05$, which is common in such a quite large sample. Therefore, the non-parametric statistic test, Spearman's Rank Order Correlation (rho) which produces a correlation coefficient that is either positive or negative and has a numerical value between -1.00 (perfect negative correlation) and +1.00 (perfect positive correlation), was deployed to measure the relationship between each construct and the level of patients' satisfaction with hospital performance and medical services.

Table 3. Tests of Normality (Kolmogorov-Smirnov \& Shapiro-Wilk)

\begin{tabular}{lllll}
\hline No & Construct & Items & Kolmogorov-Smirnov & Shapiro-Wilk \\
\hline 1 & Tangibility & $1,2,3,4,5,6,7,8 \& 27$ & .014 & .000 \\
2 & Reliability & $9,10,11,12,14,22,23,24,25,26 \& 31$ & .000 & .000 \\
3 & Responsiveness & $13,15,18,19,20 \& 21$. & .000 & .000 \\
4 & Confidence \& & $16,17,28,29,30,32,33,34,35,37,38$ & .019 & .001 \\
5 & Assurance & $\& 39$ & .044 & .001 \\
\hline
\end{tabular}

More importantly, the findings indicate that patients are generally satisfied with hospital performance and services with a mean score of 3.83 and standard deviation of .722. In addition, based on the calculated Spearman's Rho (rs) values presented in Table 4 below, the following can be concluded: 
Table 4. Spearman's Rho Coefficient Values (rs) for the Constructs of the Study

\begin{tabular}{lll}
\hline Construct & Items & Spearman's Rho Coefficient Values \\
\hline Tangibility & $1,2,3,4,5,6,7,8 \& 27$ & $0.906^{* *}$ \\
Reliability & $9,10,11,12,14,22,23,24,25,26 \& 31$ & $0.933^{* *}$ \\
Responsiveness & $13,15,18,19,20 \& 21$ & $0.935^{* *}$ \\
Confidence \& & $16,17,28,29,30,32,33,34,35,37,38 \& 39$ & $0.940^{* *}$ \\
Assurance & $36,40,41,42,43,44,45,46 \& 47$ & $0.918^{* *}$ \\
Empathy &
\end{tabular}

\subsection{Ordinal Variables Correlations}

Moving on to the correlations between the level of patients' satisfaction with hospital performance and services and the two ordinal variables, age and educational qualifications, the following can be concluded:

Table 5. Correlation Between Patients' Satisfaction and the Ordinal Variables (Age and Qualifications)

Correlations

\begin{tabular}{|c|c|c|c|c|c|}
\hline & & & Patient Satisfaction & Age & Qualifications \\
\hline \multirow{9}{*}{ Spearman's rho } & \multirow{3}{*}{$\begin{array}{l}\text { Patient } \\
\text { Satisfaction }\end{array}$} & Correlation Coefficient & 1.000 & $-.231^{*}$ & .101 \\
\hline & & Sig. (2-tailed) & 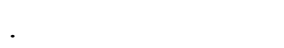 & .011 & .272 \\
\hline & & $\mathrm{N}$ & 121 & 121 & 121 \\
\hline & \multirow{3}{*}{ Age } & Correlation Coefficient & $-.231^{*}$ & 1.000 & .069 \\
\hline & & Sig. (2-tailed) & .011 & . & .451 \\
\hline & & $\mathrm{N}$ & 121 & 121 & 121 \\
\hline & \multirow{3}{*}{ Qualifications } & Correlation Coefficient & .101 & .069 & 1.000 \\
\hline & & Sig. (2-tailed) & .272 & .451 & . \\
\hline & & $\mathrm{N}$ & 121 & 121 & 121 \\
\hline
\end{tabular}

* Correlation is significant at the 0.05 level (2-tailed).

\section{Findings}

1) The values of the Spearman's Rho (rs) of all the five constructs are above 0.05 level and therefore they are significantly positively correlated.

2) The first hypothesis is supported as there is a strong positive correlation (rs 0.906) between the reliability of the medical services, as one of the quality standards, and the level of patients' satisfaction with hospital performance and services.

3) Likewise, the second hypothesis is also accepted because there is a strong positive correlation (rs 0.918) between empathy, as one of the quality standards, and the level of patients' satisfaction with hospital performance and services. This indicates that when the personnel is courteous and interested in serving patients in a professional way, this would lead to patients' satisfaction.

4) Similarly, the third hypothesis is also supported due to the strong positive correlation (rs 


\section{Macrothink}

Business and Management Horizons

ISSN 2326-0297

2019, Vol. 7, No. 1

0.940 ) found between confidence and assurance, as one of the quality standards, and the level of patients' satisfaction with hospital performance and services. This means that the more the behavior of hospital personnel instills confidence and assurance in their patients, the more the patients feel secure in dealing with the staff and become more satisfied with the service provided.

5) In addition, the fourth hypothesis is also supported due to the strong positive correlation (rs 0.906) found between tangibility, as one of the quality standards, and the level of patients' satisfaction with hospital performance and services. Therefore, it can be inferred that the more the hospital is provided with modern physical facilities and equipped with modern equipment, the more patients are satisfied.

6) In the same vein, the fifth hypothesis is also supported due to the strong positive correlation (rs 0.935 ) found between responsiveness, as one of the quality standards, and the level of patients' satisfaction with hospital performance and services. This result implies that the more the staff is interested in providing the service accurately and helping patients, the more patients' satisfaction rate increases.

7) Therefore, the more patients are satisfied with the five quality standards, the higher the quality of hospital performance and services is. Put it another way, the high levels of patients' satisfaction with the hospital performance are associated with the presence and effectiveness of the five quality standards of medical services.

8) From table 5, it can be concluded that:

9) The sixth hypothesis is rejected as there is no statistical correlation (rs .101) between educational qualification and the level of patients' satisfaction with hospital performance and services.

10) On the contrary, the seventh hypothesis is supported as there is a statistical significant negative correlation (rs -.231) between age and the level of patients' satisfaction with hospital performance and services. That is to say those elderly patients are less satisfied with the hospital performance and services than their younger counterparts. This could be attributed to the fact that elderly patients might be suffering from more chronic illnesses and therefore they could consider the medicine they get as ineffective.

However, it is worth mentioning that the strength of the relationship between the two variables, age and the level of patients' satisfaction with hospital performance and services, is considered low because it is less than .30 (Cohen, 1988). Therefore, when calculating the coefficient of determination by squaring the rs value then multiplying the result by 100 . It will look like that $-.231 *-.231 \times 100=53361$. This means that the variance shared by the two variables is just above $5 \%$ which means there is a small overlap between these two variables.

\section{Recommendations}

1) Applying quality standards will enhance the hospital competitiveness and reduce costs. 


\section{Macrothink}

Business and Management Horizons

ISSN 2326-0297

2019, Vol. 7, No. 1

2) Encouraging continuous training of the staff as means of enforcing quality standards.

3) Implementing quality standards that will make the workplace appropriate for reinforcing the spirit of teamwork, positive interaction with patients and decency in communication.

4) Paying attention to the implementation of digital health and the uses of artificial intelligence.

5) Employing programmed interaction between health organizations and patients to provide the best services and to know their needs and requests.

6) Making administrative decisions based on fulfilling patients' desires and aspirations in order to achieve their satisfaction and maintain their loyalty.

7) Making the mechanism of getting feedback from customers and patients always available in order to know the degree of their satisfaction.

8) Focusing on recruiting specialized managers with experience in management and total quality who are young and capable of using modern technology.

9) Allowing the participation of employees in making administrative decisions.

10) Affording electronic services so that appointments can be made via Smartphone.

11) Facilitating access to the medical electronic file by the patient to know his health history and help the exchange of medical information between the patient and the hospital.

12) Determining a specific number of visitors so that the hospital and its corridors are not crowded with patients.

13) Reducing the price of drugs and banning monopolizing certain brands.

14) Solving the systems problems "System Down".

15) Focusing on health insurance of elderly people, especially pensioners.

16) Designing the building originally as a hospital and avoiding renting villas or buildings.

17) Providing parking lots in a close area to the emergency department.

18) Encouraging medical research related to the standards of medical service and focusing on patients' satisfaction.

19) Benefiting from the results of scientific research which mainly aims to obtain the information needed to improve the health of individuals.

\section{References}

Abdulqader, M. (2015). Measuring The Quality Of Health Services In Government Hospitals In Sudan From The Point Of View Of Patients And Reviewers: A field study on major educational hospitals in Khartoum State. Jordanian Journal of Business Administration, 11(4). Retrieved from https://search.mandumah.com/Record/725047 


\section{Macrothink}

Business and Management Horizons

ISSN 2326-0297

2019, Vol. 7, No. 1

Al-Ahmadi, H. (2000). Doctor Professional Independence in Government Hospitals. Arab Journal of Administrative Sciences, 8(3), 315-345.

Al-Edwan, Y. (1996). Employee Satisfaction with Public Services in the Jordanian Government System: A Field Study. Yarmouk University. Erbid.

Al-Edwan, Y., \& Ahmed. A. (1997). Factors Related to Patient Satisfaction with Services Provided in Jordanian Hospitals. Al-Yarmouq Researches, 14(1), 89-101.

Al-Faraj, O. (2009). Evaluating the quality of healthcare services from the point of view of patients in higher education hospitals in Syria: A model for measuring patient satisfaction. Damascus University Journal of Economic and Legal Sciences, 25(2).

Al-Ghezawi, M. A. (2002). Total Quality Management Systems and Environment. First Edition, Dar Wael. Oman, Jordan.

Al-Hadad, S. (2006). The degree to which managers practice planning and its relation to the quality of health services. Unpublished Masters' Thesis. Academy of Graduate Studies, Department of Administration and Management. Benghazi.

Allam, S., \& Shohaib, M., A. (2001). Operations Management. Cairo.

Al-Saqaf, H. (2001). Quality Measurement in Service Companies. Youth Press, Khobar.

Al-Shenwani, S. (1996). Production Management. Alexandria Book Centre. Alexandria.

Cheng Lim, P., \& Tang, N. K. (2000). A study of patients' expectations and satisfaction in Singapore hospitals. International journal of health care quality assurance, 13(7), 290-299. https://doi.org/10.1108/09526860010378735

Christensen, C. R., Andrews, K. R., Bower, J. L., Hamermesh, R. G., \& Porter, M. E. (1982). Business policy: Text and cases. 5th ed. Homewood, IL: Richard D. Irwin.

Cohen, J. (1988). Statistical analysis for the behavioral sciences. Hillsdale: Lawrance Erlbaum, 15.

Crowder, M. J. (2017). Statistical analysis of reliability data. Routledge.

El Masry, R. B., Hamido, R. Y., \& Hilaly, H. N. (2015). Assessing the implementation of TQM in the hospitality industry in Alexandria. The Business \& Management Review, 6(4), 143-155.

Garvin, D. A. (1988). Managing quality: The strategic and competitive edge. Simon and Schuster, New York.

Goh, Y. H., Chong, V. F., \& Low, W. K. (1999). Temporal bone tumours in patients irradiated for nasopharyngeal neoplasm. The Journal of Laryngology \& Otology, 113(3), 222-228. https://doi.org/10.1017/s0022215100143622

Hamid, S. S. (2008). The impact of internal marketing as a method of human resources management on the quality of health services in the hospitals of the General Authority for 


\section{Macrothink}

Business and Management Horizons

ISSN 2326-0297

2019, Vol. 7, No. 1

Health Insurance in Greater Cairo. Accounting, Management and Insurance Journal, Faculty of Commerce, Cairo University, 60, 8-23.

Ishikawa, K. (1990). Introduction to Quality Control. 3A Corporation, Tokyo.

Jawahar, S. K. (2007). A study on out patient satisfaction at a super specialty hospital in India. Internet Journal of Medical Update, 2(2). https://doi.org/10.4314/ijmu.v2i2.39849

Kast, F. E., \& Rosenzweig, J. E. (1974). Organization and management: A systems approach. McGraw-Hill.

Khodeir. H. (2000) Total Quality Management. Dar Al-Masirah. Oman.

Mankin, D. C., \& Glueck, W. F. (1977). Strategic planning. Hospital \& health services administration, 22(2), 6-22.

Mankin. D. \& Glueck. W. (1977). "Strategic Planning," Hospital and Health Services Administration, Spring, pp. 6-22.

Matarid, N. M. (1999) Evaluation of the quality of health services provided by private hospitals. Scientific Journal of Economics and Trade, 3(1), 28-45.

Nosairat. F., \& Al-Domour., H. (2000). Similarities and differences between patients and administrators concerning the quality components of hospital services in private sector hospitals in Jordan. Journal of King Saud University - Administrative Sciences, 120(15).

Sekaran, U. (2003). Research Methods for Business: A Skill-Building Approach. 4th Edition, John Wiley \& Sons, New York.

Shaikh, B. T., \& Rabbani, F. (2005). Health management information system: a tool to gauge patient satisfaction and quality of care. Eastern Mediterranean Health Journal, Vol. 11, Nos $1 / 2$.

Slack, N., Chambers, S., \& Johnston, R. (2010). Operations management. Pearson education.

Sreenivas, T., \& Prasad, G. (2003). Patient satisfaction-a comparative study. Journal of the Academy of Hospital Administration, 15(2), 19-27.

Srour, A. S. (2003). Re-engineering Integration. Dar Al-Marikh, Jeddah.

Szilagyi, A. D., \& Sims Jr, H. P. (1974). An exploration of the path-goal theory of leadership in a health care environment. Academy of Management Journal, 17(4), 622-634. https://doi.org/10.2307/255642

Taber, K. S. (2018). The use of Cronbach's alpha when developing and reporting research instruments in science education. Research in Science Education, 48(6), 1273-1296. https://doi.org/10.1007/s11165-016-9602-2

Taguchi, G., \& Konishi, S. (1987). Taguchi Methods: Orthogonal Arrays and Linear Graphs. Tools for Quality Engineering. Dearborn, MI: American supplier Institute, pp. 35-38. 


\section{Macrothink}

Business and Management Horizons

ISSN 2326-0297

2019, Vol. 7, No. 1

Thamer, A. (2005). Marketing of Health Services. Dar Al-Basuri Scientific Publishing. Oman, Jordan.

Tiersky, E., \& Tiersky, M. (1992). The language of medicine in English. Prentice Hall Regents.

Williams, R. (1999). The Basics of Total Quality Management. Translated by Abdul-KarimAqeel. Jarir publications, Riyadh.

Wilson, A., Zeithaml, V. A., Bitner, M. J., \& Gremler, D. D. (2012). Services marketing: Integrating customer focus across the firm (No. 2nd Eu). McGraw Hill.

\section{Copyrights}

Copyright for this article is retained by the author(s), with first publication rights granted to the journal.

This is an open-access article distributed under the terms and conditions of the Creative Commons Attribution license (http://creativecommons.org/licenses/by/4.0/). 